\title{
PROCESSAMENTO AUDITIVO E POTENCIAIS EVOCADOS AUDITIVOS DE TRONCO CEREBRAL (BERA)
}

\section{Auditory precessing and auditory brainstem response (ABR)}

\author{
Marcela Pfeiffer ${ }^{(1)}$, Silvana Frota ${ }^{(2)}$
}

\begin{abstract}
RESUMO
Objetivo: verificar relação existente entre os potenciais auditivos de tronco cerebral e a avaliação comportamental do processamento auditivo. Métodos: foi realizada em um grupo de 60 meninas residentes de Paraíba do Sul na idade de nove a 12 anos com limiares tonais dentro dos padrões de normalidade e timpanometria tipo A com presença dos reflexos acústicos. Os testes utilizados para a avaliação comportamental do processamento auditivo foram: avaliação simplificada do processamento auditivo, teste de fala no ruído, teste de dissílabos alternados e teste dicótico não verbal. Após a avaliação do processamento auditivo, as crianças foram subdivididas em dois grupos, G1 (sem alteração no processamento auditivo) e G2 (com alteração no processamento auditivo) e submetidas aos potenciais auditivos de tronco cerebral. Os parâmetros utilizados na comparação dos dois grupos foram: latência absoluta das ondas I, III e V; latência interpicos das ondas I-III, I-V, III-V; diferença interaural da latência interpico I-V; e diferença interaural da latência da onda V. Resultados: foram encontradas diferenças estatísticas nos parâmetros de latência interpico das ondas I-V na orelha esquerda $(p=0,009)$, diferença interaural da latência interpico de ondas I-V $(p=0,020)$ e diferença da latência interpico de ondas I e V da orelha direita para a esquerda entre os grupos $G 1$ e G2 $(p=0,025)$. Conclusão: foi possível encontrar relação dos potenciais evocados auditivos de tronco cerebral com a avaliação comportamental do processamento auditivo nos parâmetros de latência interpico entre as ondas I e V da orelha esquerda e diferença interaural da latência interpico I-V na orelha esquerda.
\end{abstract}

DESCRITORES: Percepção Auditiva; Audiometria de Resposta Evocada; Criança

\section{INTRODUÇÃO}

Processamento auditivo (PA) refere-se ao conjunto de processos que envolvem a análise e interpretação do estímulo sonoro e pode ser definido como a série de operações mentais que o indivíduo realiza ao lidar com informações recebidas via o sentido da audição e que dependem de uma capacidade biológica inata e de experienciação no meio acústico. A desordem do processamento auditivo é a perda total ou parcial da função da análise das imagens auditivas ${ }^{1}$.

(1) Fonoaudióloga; Especialista em Audiologia pelo CEFAC - Saúde e Educação; Mestre em Fonoaudiologia pela Universidade Veiga de Almeida.

(2) Fonoaudióloga; Professora da Universidade Veiga de Almeida, UVA, Rio de Janeiro, RJ; Doutora em Distúrbios da Comunicação Humana pela Universidade Federal de São Paulo.
A avaliação das vias centrais pode ser dividida em subjetiva, dentre elas a avaliação do processamento auditivo, e a avaliação objetiva, dentre elas podem-se encontrar os potenciais evocados auditivos de tronco cerebral.

Potenciais evocados auditivos de tronco cerebral consistem em uma avaliação moderna não evasiva, objetiva da evolução neurológica do comportamento auditivo. Ou seja, consistem no registro da atividade elétrica que ocorre no sistema auditivo, da orelha interna até o córtex cerebral, em resposta a um estímulo acústico, segundo a classificação de autor ${ }^{2}$ consultado.

Em estudo relatado ${ }^{3}$, os testes comportamentais foram sensíveis para detectar disfunção de regiões cerebrais específicas, úteis em qualificar e quantificar as dificuldades auditivas vivenciadas pela criança. Adicionalmente, a função auditiva tem curso maturacional longo, alcançando padrão adulto por volta dos 16 anos ${ }^{4} \mathrm{e}$, a capacidade de reorgani- 
zação se mantém ao longo da vida ${ }^{5}$, enfatizando a necessidade de continuidade do acompanhamento fonoaudiológico desta criança.

Porém, por se tratar de duas avaliações do sistema auditivo central, acredita-se que crianças com dificuldades na avaliação subjetiva do sistema auditivo central, ou seja, avaliação comportamental do processamento auditivo, poderão apresentar alguma variação morfológica nas ondas durante a avaliação objetiva do sistema auditivo central, ou seja, potenciais auditivos de tronco cerebral. Em trabalho anterior ${ }^{6}$ constatou-se uma variação nos potenciais auditivos de curta e média latência em pessoas com alteração no processamento auditivo.

Por esta razão, este estudo teve como objetivo verificar a relação existente entre os potenciais evocados auditivos de tronco cerebral e a avaliação do processamento auditivo.

\section{MÉTODOS}

A pesquisa foi realizada com 60 crianças de nove a 12 anos de idade, todas do sexo feminino, na Clínica Centro Audiológico Regional Don Waterhouse. Todas as crianças selecionadas para a pesquisa eram alunas regulares de escola pública do município de Paraíba do Sul e internas ou semi-internas da Irmandade Nossa Senhora, irmandade esta só para meninas.

Como critério de inclusão a criança deveria apresentar respostas auditivas dentro do padrão da normalidade na avaliação auditiva periférica, composta por meatoscopia, audiometria tonal via aérea e via óssea e imitanciometria.

Para a realização da pesquisa, foram utilizados um audiômetro Amplaid 311 (dois canais), com CD player acoplado; uma cabine audiométrica; CD com exames do processamento auditivo ${ }^{6}$; um imitanciômetro diagnóstico AT 235; meatoscópio Welch Allyn; e o equipamento SmartEp, com fone de inserção do tipo E10.

Inicialmente foi aplicado o conjunto de testes do processamento auditivo nas 60 crianças do grupo amostral, com o objetivo de formar os grupos amostrais. Este conjunto de testes foi formado por, de acordo com os procedimentos descritos por autor ${ }^{7}$ : avaliação da memória sequencial verbal por meio da repetição de sequência de quatro sílabas; de memória sequencial verbal com uso de instrumentos musicais (agogô, sino, chocalho e guizo); da localização dos sons nas posições com guizo (acima, abaixo, do lado direito, do lado esquerdo e à frente da cabeça).

Para o teste monóticos utilizou-se Teste de fala com ruído. Já para a realização dos testes dicóticos foram empregados os seguintes testes: o dicótico não-verbal (escuta direcionada e atenção livre) e o dissílabos alternados (SSW). O teste diótico utilizado foi a avaliação simplificada do processamento auditivo. Os materiais utilizados para a avaliação do processamento auditivo foram os Cds com os testes propostos acoplados em um audiômetro de dois canais.

Com base na avaliação do processamento auditivo, o grupo amostral de 60 crianças foi alocado em dois grupos. O grupo 1 (G1), grupo controle, composto por 30 crianças sem distúrbio do processamento auditivo.

E o grupo 2 (G2), composto por 30 crianças com distúrbio do processamento auditivo, ou seja, que possuía alteração em pelo menos um teste do conjunto de testes do processamento auditivo, independente da categorização (codificação, organização, decodificação, ou decodificação não-verbal) e do grau (leve, moderado ou severo), propostas por autores ${ }^{8}$.

Logo após a formação dos grupos G1 e G2 foram aplicados os potenciais evocados auditivos de tronco cerebral. Os potenciais evocados auditivos de curta latência foram realizados com cliques, em uma intensidade de 70nHL, velocidade de 37.7, com um total de 1024 estímulos, em rarefação.

Os parâmetros que foram utilizados na comparação dos dois grupos, segundo a proposta de autor ${ }^{2}$, eram latência absoluta das ondas I, III e V; latência interpicos das ondas I-III, I-V e III-V; e diferença interaural da latência interpico I-V ou da latência da onda $\mathrm{V}$.

A presente pesquisa foi avaliada pelo Comitê de Ética em Pesquisa, sob $n^{\circ} 77 / 07$, tendo sido considerada como sem risco e com necessidade do consentimento livre e esclarecido.

Os resultados obtidos foram enviados para análise estatística, no qual a análise foi realizada pelos seguintes métodos:

- para comparação de variáveis numéricas entre dois grupos (caso e controle) foi utilizado o teste de Mann-Whitney (não-paramétrico) e para comparação entre quatro grupos foi realizada a Análise de Variância de Kruskal-Wallis (não-paramétrica);

- o teste dos postos sinalizados de Wilcoxon foi utilizado para verificar se existe diferença significante entre a orelha direita e esquerda.

\section{RESULTADOS}

No estudo da idade do grupo amostral dos grupos G1 e G2, observou-se que não existiu diferença significante, sendo a média de idade do grupo G1 de 10,30 anos, e do grupo G2, 10,23 anos. 
Nos resultados de latências absolutas das ondas I, III e V dos grupos G1 e G2, observou-se que não existe diferença significante entre os dois grupos nas latências absolutas de ondas I, III e V em ambas as orelhas.

$\mathrm{Na}$ Figura 1 encontram-se as latências interpicos de ondas I-III, I-V e III-V dos grupos G1 e G2, com a média, desvio padrão (DP), mediana e análise estatística da orelha direita.

Observou-se que, não existe diferença significante entre os dois grupos nas latências interpicos de ondas I-III, I-V e III-V na orelha direita.

$\mathrm{Na}$ Figura 2 mostram-se as latências interpicos de ondas I-III, I-V e III-V dos grupos G1 e G2, com a média, desvio padrão (DP), mediana e análise estatística da orelha esquerda.

Observou-se que o grupo G2 apresentou latência interpico I-V significantemente maior do que o grupo G1. Nos demais valores não existem diferença significante nas latências interpicos dos dois grupos na orelha esquerda.

Nas medidas de latência absoluta e latências interpicos segundo o grau do grupo G2 (leve, moderado, severo) em relação ao grupo G1, observou-se que não houve diferenças significantes em ambas as orelhas.

Verificou-se uma tendência nos valores de latência interpicos de ondas I-V na comparação do grau do grupo G2 com o grupo G1 (controle) na orelha esquerda.

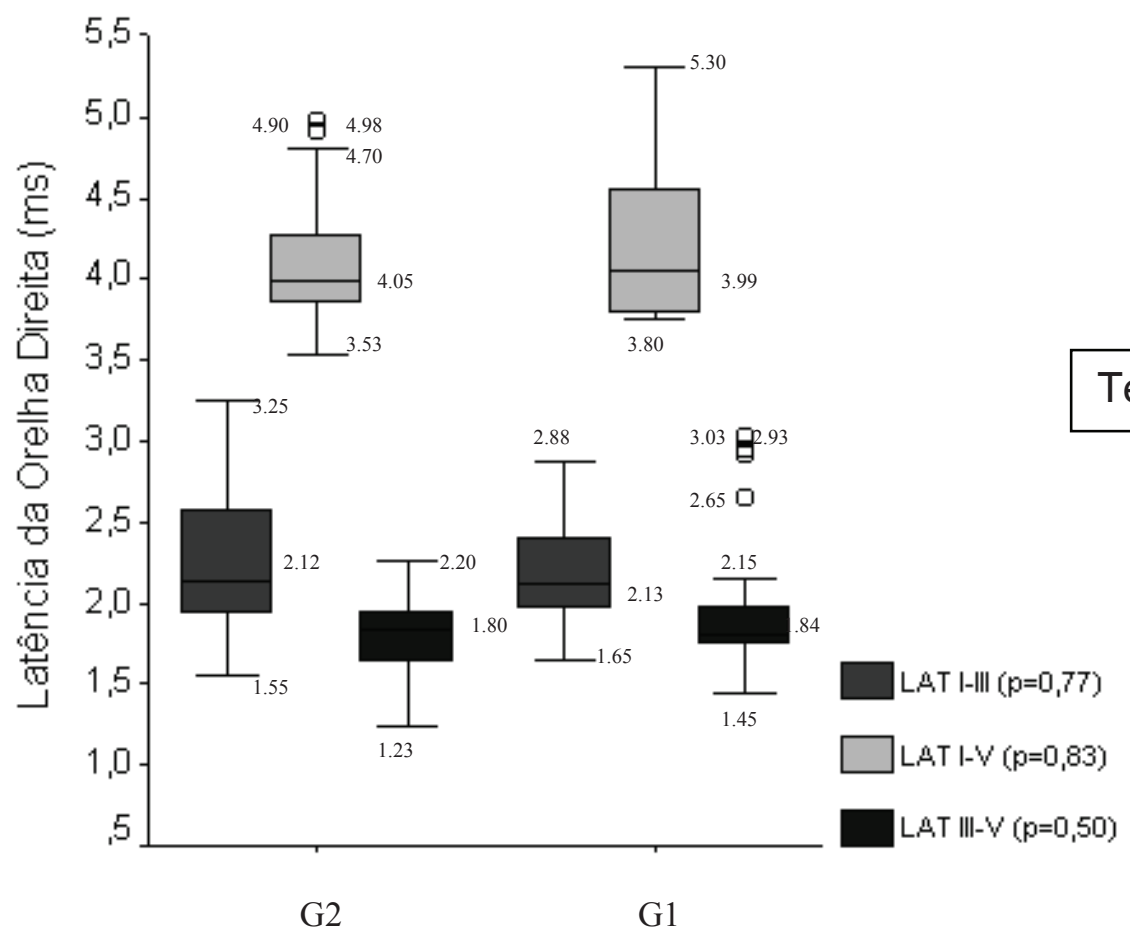

Figura 1 - Latência interpicos de ondas I-III, I-V e III-V dos grupos G1 e G2 na orelha direita
Observou-se, também, que não existe diferença significante na latência $V$ e na latência I-V da orelha direita para a esquerda no grupo G1. Entretanto, foi possível verificar diferença significante na latência I-V da orelha direita para a esquerda no grupo G2, sendo maior para a esquerda. $E$, além disso, observou-se, demonstrado na Tabela 1, que o grupo G2 apresentou diferença da latência interpico de ondas I e $\mathrm{V}$ da orelha direita para a esquerda significantemente maior que o grupo $\mathrm{G} 1$.

\section{DISCUSSÃO}

As respostas dos potenciais auditivos de tronco cerebral são de grande importância na avaliação da integridade do tronco cerebral auditivo. Segundo autor $^{6}$, a aplicação dos potenciais evocados auditivos possibilita a avaliação da sensibilidade auditiva de recém-nascidos, crianças ou mesmo adultos nos quais não tenha sido possível a realização de um teste confiável, ou nos casos em que é impossível a utilização de outro método auditivo convencional.

Apesar de autor consultado ${ }^{10}$ ter afirmado que os potenciais evocados auditivos de tronco cerebral é uma avaliação sensível para detectar a perda auditiva, acredita-se que é possível encontrar alterações nos potenciais evocados auditivos em crianças com alteração do processamento auditivo.
Teste de Mann-Whitney 


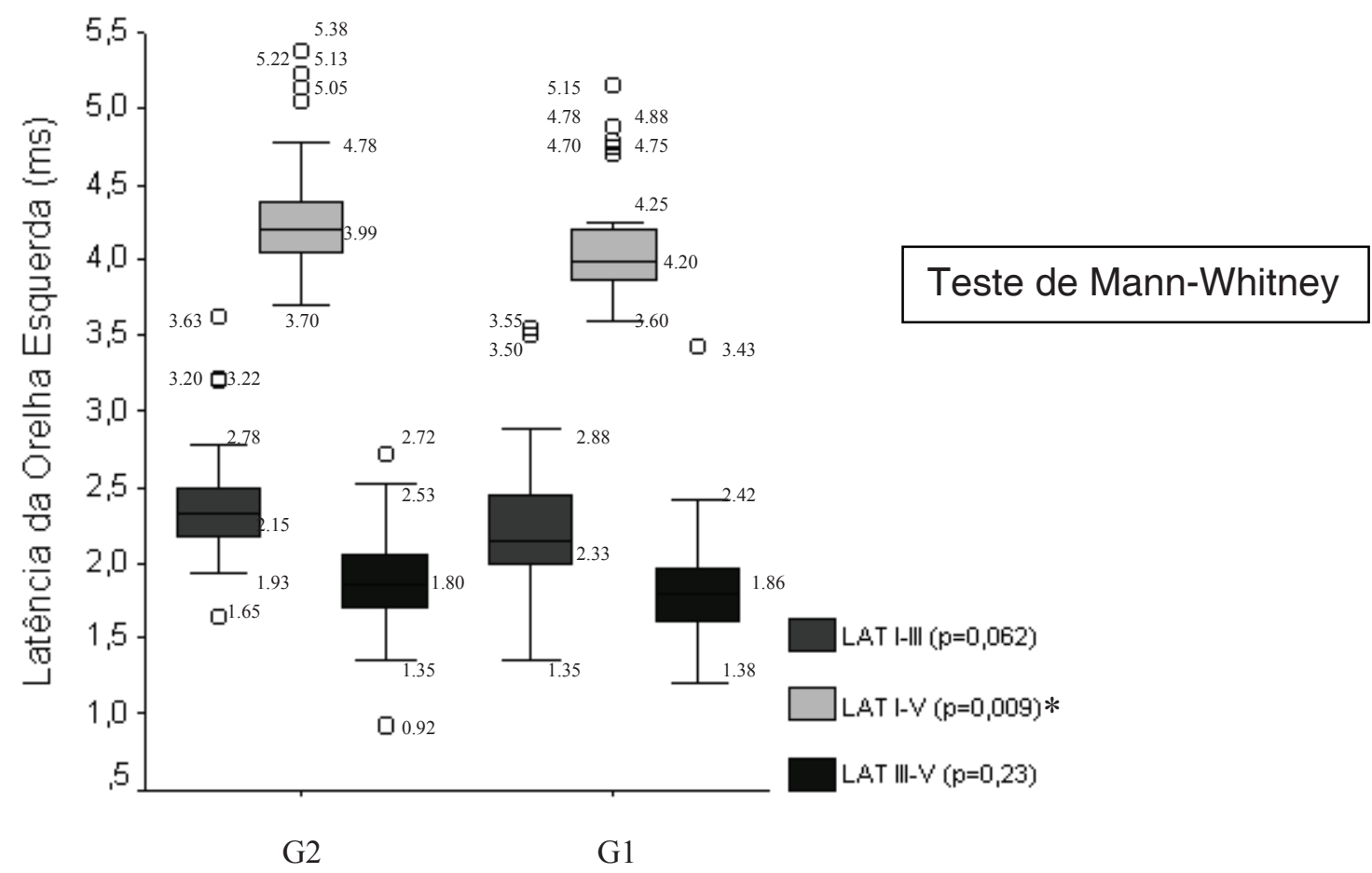

Figura 2 - Latência interpicos de ondas I-III, I-V e III-V dos grupos G1 e G2 na orelha esquerda

Tabela 1 - Análise da diferença entre orelhas da latência absoluta de onda $\mathbf{V}$ e latência interpico de ondas I-V para o grupo G2

\begin{tabular}{lcccc}
\hline Medida & Média & DP/EP & Mediana & p valor \\
\hline LAT V - OD & 5,799 & 0,344 & 5,71 & \\
LAT V - OE & 5,925 & 0,354 & 5,87 & \\
DIF LAT V (D-E) & $-0,125$ & 0,082 & $-0,13$ & 0,085 \\
INTER I-V - OD & 4,097 & 0,383 & 3,99 & \\
INTER I-V - OE & 4,294 & 0,426 & 4,20 & \\
DIF INTER I-V (D-E) & $-0,197$ & 0,092 & $-0,17$ & $0,020^{*}$ \\
\hline
\end{tabular}

Por esta razão, nesta pesquisa foram incluídas crianças que apresentaram normalidade na audiometria tonal limiar e na imitanciometria, classificados por autores ${ }^{11,12}$, com o objetivo de evitar que perdas auditivas periféricas influenciassem nos resultados de latência dos potenciais evocados auditivos de tronco cerebral.

A pesquisa foi realizada com crianças entre nove e 12 anos de idade, pois as respostas dos testes escolhidos no conjunto da avaliação comportamental do processamento auditivo, proposta por autor ${ }^{7}$, possuem a mesma padronização para esta faixa etária.

Nos resultados da avaliação do processamento auditivo, a dificuldade mais encontrada nas crianças do grupo amostral G2 foi a de categorização do tipo de decodificação e de grau moderado.
A categorização denominada do tipo decodificação não verbal, definida por autor ${ }^{8}$, avaliada no teste dicótico não-verbal, foi observada em algumas crianças com processamento auditivo alterado (37\%), mas não foi a categorização mais encontrada.

Os mecanismos fisiológicos encontrados alterados foram: reconhecimento de sons verbais em escuta dicótica e reconhecimento de sons não verbais em escuta dicótica, segundo a classificação de autor consultado ${ }^{13}$.

Os achados podem ser associados ao que autor ${ }^{14}$ afirmou que existem dois tipos de avaliações das vias auditivas centrais, a avaliação objetiva, com os potenciais evocados auditivos, e a avaliação subjetiva, com a avaliação comportamental do proces- 
samento auditivo, e por esta razão, crianças com alteração do processamento auditivo podem apresentar potenciais auditivos de tronco cerebral, já que ambas avaliações pesquisam a mesma estrutura.

Além disso, os resultados encontrados podem ser associados à afirmativa de autor ${ }^{15}$ quando dissera que o distúrbio de o processamento auditivo ser uma desordem resultante de uma função auditiva central deficiente, pelo menos em nível de tronco cerebral.

Os resultados encontrados também mostram que a alteração do processamento auditivo pode decorrer de outros fatores além de dissincronia neural, como afirmara autor ${ }^{16}$, pois não foi possível encontrar diferença estatisticamente significante entre os grupos G1 e G2 na maior parte dos parâmetros estudados neste estudo.

Segundo a descrição de literatura consultada ${ }^{17}$, os potenciais evocados auditivos de tronco cerebral são avaliações da integridade e evolução das vias auditivas. Já outro estudo ${ }^{18}$ afirma ser um instrumento cujo principal objetivo é complementar os procedimentos audiológicos de rotina, auxiliando no diagnóstico de problemas auditivos difíceis de serem avaliados de forma confiável.

Por esta razão acredita-se que os potenciais evocados auditivos de tronco cerebral podem ser influenciados pela dificuldade do sistema auditivo, mesmo que seja central, e estar diretamente ligado ao desenvolvimento das habilidades auditivas, concordando assim com autores consultados 8,16 .

As ondas formadas pelos potenciais evocados auditivos de tronco cerebral são geradas pela ativação sequencial e sincrônica das fibras nervosas ao longo da via auditiva, e por esta razão pode ser alterada de acordo com a maturação da via auditiva, mesmo que seja acima do tronco cerebral.

Essa interferência da maturação auditiva acima do tronco cerebral nos potenciais evocados auditivos de tronco cerebral pode estar associada aos achados do estudo ${ }^{16}$, já que crianças com alterações nos testes comportamentais do processamento auditivo, testes que avaliam mecanismos fisiológicos encontrados até nível de córtex cerebral, apresentaram uma diferença estatisticamente significante nas latências interpicos I-V na orelha esquerda.

Pode-se encontrar respostas auditivas da oreIha direita melhores que as respostas da orelha esquerda, segundo afirmações de autor ${ }^{19}$ consultado. Essa afirmativa pode ser associada a este estudo já que foram encontradas diferenças estatisticamente significantes apenas na orelha esquerda.

Conforme definido antes, o processamento auditivo é a percepção em nível central de estímulos auditivos captados perifericamente, porém os resultados mostram mesmo com o processamento auditivo alterado pode-se encontrar resultados de potenciais evocados auditivos de tronco cerebral normais, em relação aos valores dos parâmetros de latência.

Apesar de ter-se encontrado resultados significantes nas respostas dos potenciais evocados auditivos de tronco cerebral, não foi possível encontrar uma relação clara com a avaliação comportamental do processamento auditivo, já que não houve diferença significante em alguns fatores dos potenciais evocados auditivos de tronco cerebral entre os dos grupos amostrais.

Esses achados, então, podem concordar com estudo ${ }^{20}$ quando associara outros exames à avaliação do processamento auditivo, inclusive os potenciais evocados auditivos de média e longa latência. Então, com isso, pode-se pensar na possibilidade dos potenciais auditivos evocados de média e longa latência serem mais eficazes no estudo do processamento auditivo.

Estes achados, por outro lado, não podem contestar autor ${ }^{21}$ quando afirma que encontradas alterações de latência e amplitude dos potenciais evocados auditivos de tronco cerebral durante a idade de neonato, e com uma intervenção precoce podem propiciar um prognóstico melhor para esta criança.

Além de tentar melhorar seu prognóstico quanto à aquisição de linguagem, um fator bastante presente em crianças que necessitam da avaliação do processamento auditivo, pode-se aumentar as possibilidades de inseri-las no meio social, concordando com o que afirmara autores consultados ${ }^{22}$.

\section{CONCLUSÃO}

Foi possível encontrar relação entre os potenciais evocados auditivos de tronco cerebral e a avaliação comportamental do processamento auditivo através dos parâmetros de latência interpico entre as ondas I e $V$ da orelha esquerda e de diferença interaural da latência interpico I-V na orelha esquerda, pois crianças com processamento auditivo alterado apresentaram os valores destas latências maiores do que crianças sem alteração no processamento auditivo. 


\section{ABSTRACT}

Purpose: to investigate the correlation of auditory brainstem response (ABR) and behavioral auditory processing evaluation. Methods: sixty girls, from Paraíba do Sul, ranging from 9 to 12-year-old were evaluated. In order to take part in the study all of them should have normal hearing thresholds, type A tympanograms and presence of acoustic reflexes. Behavioral auditory processing evaluation consisted of: sound localization, memory for verbal and non verbal sounds in sequence, speech in noise test, SSW test and nonverbal dichotic test. After the behavioral testing children were divided into two groups, G1 ( without auditory processing disorders) and G2 (with auditory processing disorders of any degree and categorization) and have undergone the register of auditory brainstem response (ABR). The groups were compared using absolute latencies of Waves I, III, V and interpeak latencies I-III, III-V, I-V and interaural difference of Wave V latency. Results: our results indicated statistical significant differences considering interpeak latency in the left ear $(p=0.009)$, in right and left ears considering interpeak latencies for $\mathrm{G} 2(\mathrm{p}=0.02)$ and in interpeak latencies of waves I and $\mathrm{V}$ considering right and left ears between the groups $\mathrm{G} 1$ and $\mathrm{G} 2(\mathrm{p}=0.025)$. Conclusion: significant differences were observed comparing behavioral evaluation and $A B R$ considering interpeak latency of waves I and $V$ in the left ear and interaural difference I-V in the left ear.

KEYWORDS: Auditory Perception; Audiometry, Evoked Response; Child

\section{REFERÊNCIAS}

1. Andrade AN, Gil D, Schiefer AM, Pereira LD. Avaliação comportamental do processamento auditivo em indivíduos gagos. Pró-Fono. 2008; 20(1):43-8.

2. Figueiredo MS, Costa Júnior NP. Potenciais evocados auditivos de tronco encefálico (ABR). In: Figueiredo MS. Emissões otoacústicas e BERA. São Paulo: Pulso; 2003. p. 85-98.

3. Elias KMIF, Santos MFC, Ciasca SM, MouraRibeiro MVL. Processamento auditivo em criança com doença cerebrovascular. Pró-Fono. 2007; 19(4):393-400.

4. Bishop DV, McArthur GM. Individual differences in auditory processing in specific language impairment: a follow-up study using event-related potentials and behavioral thresholds. Cortex. 2005; 41(3):327-41.

5. Bütefisch $\mathrm{CM}$. Plasticity in the human cerebral cortex: lessons from the normal brain and from stroke. Neuroscientist. 2004 Apr; 10(2):163-73.

6. Sens PM, Almeida CIR. Participação do cerebelo no processamento auditivo. Rev Bras Otorrinolaringol. 2007; 73(2):266-70.

7. Pereira LD, Schochat E. Processamento auditivo central: manual de avaliação. São Paulo: Lovise; 1997.

8. Pereira LD. Sistema auditivo e desenvolvimento das habilidades auditivas. In: Ferreira LP, Befi-LopesO DM, Limongi SCO. Tratado de fonoaudiologia. São Paulo: Roca; 2004. p. 547-52.
9. Sousa LCA, Rodrigues LS, Piza MRT, Ferreira DR, Ruiz DB. Achado ocasional de doenças neurológicas durante a pesquisa da surdez infantil através do BERA. Rev Bras Otorrinolaringol. 2007; 73(3):424-8.

10. Stueve MP, O'Rouke C. Estimation of hearing loss in children: comparison of auditory steadystate response, auditory brainstem response, and behavioral test methods. Am J Audiol. 2003. 12(2):125-36.

11. Silva IMC, Feitosa MAG. Audiometria de alta frequência em adultos jovens e mais velhos quando a audiometria convencional é normal. Rev Bras Otorrinolaringol. 2006; 72(5):665-72.

12. Feniman MR, Souza AG, Jorge JC, Lauris JRP. Achados otoscópicos e timpanométricos em lactentes com fissura labiopalatina. Rev Bras Otorrinolaringol. 2008; 74(2):248-52.

13. Pereira LD, Cavadas M. Processamento auditivo. In: Frota S. Fundamentos em fonoaudiologia: audiologia. Rio de Janeiro: Guanabara; 2003: 141-56

14. Chermak GD, Musiek FE. Auditory training: principles and approaches for remediating and managing auditory processing disorders. Sem Hear. 2002; 23(4):297-308.

15. Rosen S. "A riddle wrapped in a mystery inside an enigma": defining central auditory processing disorder. Am J Audiol. 2005; 14(2):139-42.

16. Neves IF, Schochat E. Maturação do processamento auditivo em crianças com e 
sem dificuldades escolares. Pró-Fono. 2005; 17(3):311-20.

17. Rezende MSM, lório MCM. Potenciais evocados auditivos: estudo com indivíduos portadores de lúpus eritematoso sistêmico. Rev Bras Otorrinolaringol. 2008; 74(3):429-39.

18. Pinto FR, Matas CG. Comparação entre limiares de audibilidade e eletrofisiológico por estímulo tone burst. Rev Bras Otorrinolaringol. 2007; 73(4):513-22.

19. Marotta RMB, Quintero SM, Marone SAM. Avaliação do processamento auditivo por meio do teste de reconhecimento de dissílabos em tarefa dicótica SSW em indivíduos com audição normal e ausência do reflexo acústico contralateral. Rev Bras Otorrinolaringol. 2002; 68(2):254-61.

20. Zalcman TE, Schochat E. A eficácia do treinamento auditivo formal em indivíduos com transtorno de processamento auditivo. Rev Soc Bras Fonoaudiol. 2007; 12(4):310-4.

21. Fuess VLR, Bento RF, Silveira JAM. Delay in maturation of the auditory pathway and its relationship to language acquisition disorders. Ear Nose Throat J. 2002; 81(10): 706-12.

22. Person OC, Marone MR, Jardim M, Rapoport PB. A utilização dos potenciais evocados auditivos como método diagnóstico em medicina. Arq Médico ABC. 2005; 30(1):5-10.

RECEBIDO EM: 06/10/2008

ACEITO EM: 09/02/2009

Endereço para correspondência:

Marcela Pfeiffer

Rua do Imperador, 319 ap. 401

Petrópolis - RJ

CEP: 25620-002

E-mail: marcela.pfeiffer@gmail.com 